\title{
Seroconversion Following SARS-CoV-2 Infection or Vaccination in Pediatric IBD Patients
}

\section{Elizabeth A. Spencer, MD,* ED Eyal Klang, MD, ${ }^{\dagger}$ Michael Dolinger, MD, MBA,* Nanci Pittman, MD,* and Marla C. Dubinsky, MD*}

From the *Department of Pediatrics, Susan and Leonard Feinstein Inflammatory Bowel Disease Clinical Center, Mount Sinai Hospital, New York, NY, USA

†'Institute for Healthcare Delivery Science, Department of Population Health Science and Policy, Icahn School of Medicine at Mount Sinai, New York, NY, USA

Address correspondence to: Elizabeth Spencer, MD; One Gustave L. Levy Place, Box 1656, New York, NY 10029, USA (elizabeth.spencer@mssm.edu).

Key words: inflammatory bowel disease, Crohn's disease, ulcerative colitis, IBD-unspecified, SARS-CoV2, COVID-19, vaccination, antibody, pediatric

\section{Introduction}

Protective immunity to SARS-CoV-2, either naturally induced by infection or artificially induced or augmented by vaccination, is vital to reducing the transmission of SARSCoV-2 and the burden of severe coronavirus disease 2019 (COVID-19). Data on the impact of immunomodulatory therapies used to treat inflammatory bowel disease (IBD) on both types of protective immunity have been scant, with initial reports of attenuation of natural immunity and vaccine efficacy by TNF antagonists. ${ }^{1,2}$ However, 2 doses of COVID-19 vaccine or a single dose in those with a history of SARS-CoV-2 infection have both been shown to induce seroconversion in a large majority of patients. ${ }^{2,3}$ There is little data on seroconversion in pediatric IBD, a population that may have distinct immunologic responses to SARS-CoV-2, given that increased age has been associated with lower antibody concentrations after COVID-19 vaccination. ${ }^{2}$ Understanding seroconversion is particularly important as vaccination becomes more widely available in pediatrics with the recent emergency use approval of BNT162b2 (Pfizer-BioNTech) down to 12 years of age. ${ }^{4}$ We therefore sought to evaluate and compare serologic responses to SARS-CoV-2 infection and vaccination in a pediatric IBD cohort.

\section{Methods}

We conducted a retrospective chart review of all IBD patients younger than 21 years old in whom a SARS-CoV-2 immunoglobulin $\mathrm{G}$ ( $\mathrm{IgG}$ ) antibody assay was performed between April 2020 and May 2021 at our tertiary care center. The COVID-SeroKlir (Kantaro Biosciences, LLC, New York, NY) semiquantitative SARS-CoV-2 IgG antibody assay, an enzyme-linked immunosorbent assay (ELISA) measuring IgG antibody to the full-length SARS-CoV-2 spike protein with emergency use authorization, was routinely collected at infusion and outpatient clinic visits. ${ }^{5}$ The study was approved by the Mount Sinai institutional review board.
Electronic medical records were reviewed, and data were collected on demographics, IBD location and behavior (Paris classification), ${ }^{6}$ exposure(s) to SARS-CoV-2, and history of SARS-CoV-2 infection/symptoms. Indication for antibody testing and titer levels, which were described as high titer or strongly positive ( $\geq 960$ titer or $>40 \mathrm{AU} / \mathrm{mL}$ ), moderately positive (320-960 titer or 16-39 AU/mL), weakly positive (80-160 titer or 5-15 AU/mL), and negative $^{5}$ were recorded.

Patients meeting World Health Ogranization (WHO) criteria for confirmed (laboratory confirmation of COVID-19 infection) or probable COVID-19 (either meeting defined clinical criteria with contact with a probable or confirmed case of COVID-19 or recent onset of anosmia/ageusia without an identified cause) were classified as having prior COVID-19 infection.?

Standard descriptive statistics, including frequency for categorical variables and median (interquartile range [IQR]) for continuous variables, were calculated unless otherwise stated. Univariate analyses were performed using Fisher exact and $\chi^{2}$ test for categorical variables and MannWhitney and Spearman Rank Coefficient for continuous variables where appropriate. Statistical analyses were performed using R 3.6.3 (The R Foundation for Statistical Computing, 2018) and SAS OnDemand for Academics 3.8 (SAS Institute Inc., Cary, NC, 2020). A $P$ value $\leq .05$ was considered statistically significant.

\section{Results}

One or more SARS-CoV-2 IgG antibody assay(s) was performed in 340 pediatric patients; $15 \%(n=51)$ were confirmed or probable for COVID-19; $2 \%(n=7)$ were suspected for COVID-19; $16 \%(n=54)$ were exposed to or had close contact with SARS-CoV-2 infection without clinical symptoms; $61 \%(n=208)$ did not have any prior symptoms or exposures; and $6 \%(n=20)$ had a history of COVI-19 vaccination. In the 51 patients with confirmed or probable 
COVID-19, 90\% had seroconversion (Supplementary Table 1). Of the 5 patients without evidence of seroconversion, 3 had confirmed and 2 had probable COVID-19. Those that did not seroconvert were similar in age to the rest of the postinfection cohort (median 20 [17-20] years old, $P=.15$ ), with a trend to a higher proportion of patients with UC/ IBD-U $(60 \%, P=.07) ; 4$ patients $(80 \%)$ were on a biologic therapy ( 3 infliximab, 1 ustekinumab), and the remaining patient was on 5-aminosalicylate (5-ASA). There was a significantly longer time interval between infection and titer level measurement in those with a negative titer (negative, 257 [167-340] days; positive, 112 [41-180] days; $P=.03$ ); however, titer level was not correlated with time from infection in the entire postinfection cohort $(P=-0.06, P=.74)$.

Within the $16 \%$ of patients with exposure to SARSCoV-2 without clinical symptoms, $23(43 \%)$ had a positive SARS-CoV-2 antibody assay. There were no identified clinical characteristics associated with seroconversion in the group exposed to SAR-CoV-2 without clinical symptoms (Supplementary Table 2).

Twenty patients had antibody testing after vaccination (Table 1). All patients seroconverted following vaccination, and all patients receiving mRNA vaccination had high titer levels. The patient who received a single dose of JNJ78436735 (Johnson \& Johnson) had a moderate titer level. All but $1(95 \%)$ of those receiving a 2 -series mRNA vaccination had completed both vaccinations in the series; the single patient with an assay performed after only 1 dose of BNT162b2, with no prior history of SARS-CoV-2 infection, seroconverted. Titer level was not significantly associated with type of biologic or small molecule therapy (Figure 1); patients receiving mRNA-1273 (NIH-Moderna) did have significantly higher titer levels compared to BNT162b2 and JNJ-78436735 $(P=.005)$.

\section{Discussion}

Herein we report robust serologic antibody responses to SARS-CoV-2 infection and COVID-19 vaccination in a pediatric IBD cohort. All of our patients seroconverted after vaccination even in the setting of biologic and small molecule usage, which was similar to the findings in an adult IBD study published out of our center. ${ }^{3}$ Moreover, nearly all (90\%) of our patients had seroconversion following SARS-CoV-2 infection, which was higher than the rates seen in Kennedy et al, suggesting improved postinfection seroconversion in pediatrics. ${ }^{1}$ The high titer levels achieved in a large number of those who seroconverted are thought to confer protection; however, the association with elapsed time from SARS-CoV-2 exposure to negative level warrants continued investigation into the longevity of the protection conferred and more detailed cataloging of the complexities of the immunoprotective response beyond $\mathrm{IgG}$ antibodies. Although we are limited by our small sample size and variable times to assay, this study provides important reassurances to pediatric gastroenterologists, patients, and families and lends further support to expert consensus recommendations for vaccination of IBD patients. ${ }^{8}$
Table 1. Clinical characteristics of pediatric patients with IBD who received COVID vaccination.

\begin{tabular}{|c|c|}
\hline \multirow[t]{2}{*}{ Clinical Characteristic } & \multirow{2}{*}{$\begin{array}{l}\text { Vaccination }+/ \\
\text { Prior Infection } \\
n=20\end{array}$} \\
\hline & \\
\hline \multicolumn{2}{|l|}{ Demographics } \\
\hline Male, N (\%) & $12(60)$ \\
\hline Age (years), Median (IQR) & $18(17-20)$ \\
\hline \multicolumn{2}{|l|}{ Vaccination Type } \\
\hline BNT162b2 (Pfizer-BioNTech) & $14(70)$ \\
\hline mRNA-1273 (NIH-Moderna) & $5(25)$ \\
\hline JNJ-78436735 (Johnson \& Johnson) & $1(5)$ \\
\hline \multicolumn{2}{|l|}{ IBD Subtype, N (\%) } \\
\hline Crohn's Disease & $15(75)$ \\
\hline Ulcerative colitis & $5(25)$ \\
\hline \multicolumn{2}{|l|}{ Age of Diagnosis, N (\%) } \\
\hline Diagnosis $<17$ years & $17(85)$ \\
\hline \multicolumn{2}{|l|}{ Crohn's Disease Location } \\
\hline Ileal & $4(29)$ \\
\hline Colonic & $1(7)$ \\
\hline Ileocolonic & $8(57)$ \\
\hline Isolated Upper Tract & $1(7)$ \\
\hline \multicolumn{2}{|l|}{ Crohn’s Disease Behavior, N (\%) } \\
\hline Non-penetrating, nonstricturing & $11(85)$ \\
\hline Stricturing & $1(8)$ \\
\hline Penetrating & $0(0)$ \\
\hline Stricturing and Penetrating & $1(8)$ \\
\hline Perianal Disease & $2(15)$ \\
\hline \multicolumn{2}{|l|}{ Ulcerative Colitis/IBD-U, N (\%) } \\
\hline Proctitis & $0(0)$ \\
\hline Left-sided & $1(20)$ \\
\hline Extensive/pancolitis & $4(80)$ \\
\hline \multicolumn{2}{|l|}{ IBD Therapy, N (\%) (Supplementary Figure 1B) } \\
\hline Biologic Therapy & $19(95)$ \\
\hline Infliximab & $7(37)$ \\
\hline Adalimumab & $2(11)$ \\
\hline Ustekinumab & $10(53)$ \\
\hline Vedolizumab & $0(0)$ \\
\hline Tofacitinib & $2(10)^{\mathrm{c}}$ \\
\hline \multicolumn{2}{|l|}{ Disease Activity, N (\%) } \\
\hline $\begin{array}{l}\text { Clinical remission a at time of } \\
\text { vaccination }\end{array}$ & $17(89)$ \\
\hline \multicolumn{2}{|l|}{ SARS-CoV-2 Antibody Testing } \\
\hline Antibody positive, $\mathrm{N}(\%)$ & $20(100)$ \\
\hline $\begin{array}{l}\text { Median (IQR) time from last vaccination to } \\
\text { titer (days) }\end{array}$ & $29(14-37)^{\mathrm{b}}$ \\
\hline High titer, $N(\%)$ & $18(95)^{\mathrm{b}}$ \\
\hline History of Infection, N (\%) & $5(25)$ \\
\hline
\end{tabular}

${ }^{a}$ Clinical remission: partial Mayo Score $<2$ or Harvey-Bradshaw Index $<4$ ${ }^{\mathrm{b} S}$ Single patient with qualitative titer only available

${ }^{\mathrm{c}}$ One patient was on combination ustekinumab and tofacitinib 


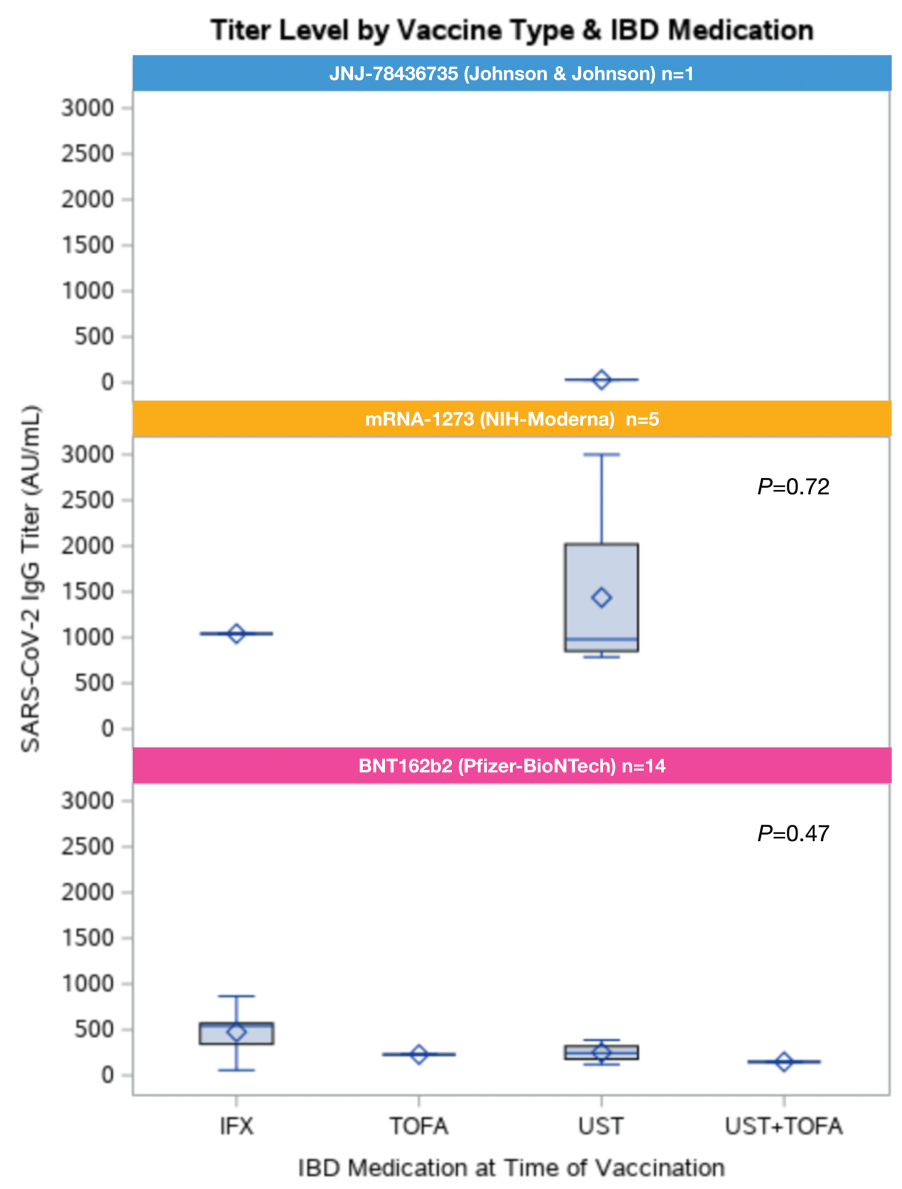

Figure 1. Titer level by IBD therapy and vaccine type. Abbreviations: IFX, infliximab; TOFA, tofacitinib; UST, ustekinumab.

\section{Supplementary Data}

Supplementary data is available at Inflammatory Bowel Diseases online.

\section{Acknowledgments}

The authors wish to thank the pediatric gastroenterologists at the Mount Sinai IBD Center and Randa Samaha, FNP.

\section{Funding}

E.A.S. is supported by a National Institutes of Health T32 grant (5T32GM082773-14).

\section{Conflicts of Interest}

M.C.D. is a consultant for Janssen, Abbvie, UCB, Takeda, Pfizer, Prometheus Labs, Genentech, Salix, Celgene Research Support, Takeda, Pfizer, and Janssen. M.T.D. is a consultant for Neurologic Corp., a subsidiary of Samsung Electronics Co., Ltd. The remaining authors disclose no conflicts.

\section{References}

1. Kennedy NA, Goodhand JR, Bewshea C, et al. Anti-SARS-CoV-2 antibody responses are attenuated in patients with IBD treated with infliximab. Gut. 2021;70:865-875.
2. Kennedy NA, Lin S, Goodhand JR, et al. Infliximab is associated with attenuated immunogenicity to BNT162b2 and ChAdOx1 nCoV-19 SARS-CoV-2 vaccines in patients with IBD. Gut: 2021. [published online ahead of print]. doi:10.1136/ gutjnl-2021-324789

3. Wong SY, Dixon R, Pazos VM, Gnjatic S, Colombel JF, Cadwell K. Serological response to mRNA COVID-19 vaccines in IBD patients receiving biological therapies. Gastroenterology. 2021;161:715718.e4. doi:10.1053/j.gastro.2021.04.025.

4. U.S. Food and Drug Administration. Coronavirus (COVID-19) update: FDA authorizes Pfizer-BioNTech COVID-19 vaccine for emergency use in adolescents in another important action in fight against pandemic [press release]; 2021. Available at: https:// www.fda.gov/news-events/press-announcements/coronaviruscovid-19-update-fda-authorizes-pfizer-biontech-covid-19-vaccineemergency-use. Accessed May 15, 2021.

5. Amanat F, Stadlbauer D, Strohmeier S, et al. A serological assay to detect SARS-CoV-2 seroconversion in humans. Nat Med. 2020;26:1033-1036.

6. Levine A, Griffiths A, Markowitz J, et al. Pediatric modification of the Montreal classification for inflammatory bowel disease: the Paris classification. Inflamm Bowel Dis. 2011;17:13141321.

7. World Health Organization. Who COVID-19: Case Definitions. World Health Organization; 2020: December.

8. Siegel CA, Melmed GY, McGovern DP, et al. SARS-CoV-2 vaccination for patients with inflammatory bowel diseases: recommendations from an international consensus meeting. Gut. 2021;70:635-640. 